\title{
Les facteurs déterminants de la réception de transferts de fonds des migrants et leur impact sur la pauvreté en Albanie
}

\author{
Mathias Lerch, Philippe Wanner, Neuchâtel
}

\section{Introduction}

Alors que les coûts de la migration pour les pays du Sud sont généralement mentionnés (p.ex. fuite des cerveaux), peu d'attention est actuellement portée aux bénéfices, en termes de développement économique et social, que ces pays tirent de «l'exportation» de leur main d'œuvre. Outre les transferts de capitaux humains et sociaux, les bénéfices les plus immédiats pour les pays sources d'immigration sont les transferts de fonds rapatriés par les travailleurs émigrés.

Le montant total de ces transferts en direction des pays en voie de développement a été évalué, pour la période comprise entre 1991 et 1999, à 450 milliards de dollars (comprenant les flux financiers d'un pays du Sud à un autre pays du Sud), ce qui représente $15 \%$ de l'ensemble des flux financiers en direction de ces pays durant la même période (GAmmeltopf 2002). Dans une perspective d'aide au développement, ces transferts représentent la deuxième source de devises pour les Pays en Voie de Développement (PVD) après les investissements étrangers directs - et leur montant excède de $20 \%$ celui des aides financières directes (GAmmeltopf 2002). Outre leur stabilité face à la conjoncture, les transferts de fonds présentent l'intérêt, dans une optique d'aide au développement, de toucher directement les groupes les plus pauvres (Ratha 2004). Pour l'Albanie, on considère que les transferts de fonds ont atteint 606,8 millions de dollars en 2002, représentant 12,9\% du Produit National Brut (PNB) (contre $20 \%$ en 1993). L'Albanie est d'ailleurs en tête des pays en développement pour cet indicateur, les pays à revenu moyen inférieur étant, en effet, les principaux bénéficiaires de ces fonds. Ces chiffres ne prennent pas en compte les capitaux envoyés à travers des canaux officieux, estimés à $60 \%$ de l'ensemble des transferts (URIÇı \& Gedeshi 2003).

Parallèlement à l'évolution de cet apport de devises, le phénomène migratoire est devenu difficilement maîtrisable et gagne progressivement en complexité et en diversité. Les efforts entrepris par les pays industrialisés pour contrôler les frontières nationales sont dorénavant liés à une volonté de coopérer avec les pays d'émigration, afin de soulager la pression des migrations, notamment par l'aide au développement. Or, le constat d'échec à ce propos laisse progressive- ment sa place à de nouveaux concepts davantage axés sur l'empowerment des pays concernés. Etant donné le caractère dynamique et cyclique de la migration contemporaine, de nombreuses politiques occidentales discutent de l'établissement de programmes valorisant leurs transferts de fonds; le migrant devient ainsi un acteur du développement.

Inspirées de ces préoccupations politiques, la majorité des études sur les transferts de fonds (ou, pour utiliser le terme consacré, les remittances) se concentrent sur leur impact macroéconomique. Cet impact est étroitement déterminé par le profil des bénéficiaires de ces transferts et par l'usage qu'ils en font: p.ex. dépenses de la vie quotidienne, investissements. L'identification et la description de la population bénéficiaire permettent dès lors de comprendre les relations entre fonds transférés et développement, et de favoriser les projets de valorisation de ces fonds.

Cette description est l'objectif de la présente analyse, qui vise à montrer les caractéristiques démographiques, socio-économiques, migratoires et géographiques des ménages albanais bénéficiant de remittances. A l'aide d'un modèle statistique, nous mettons en évidence, dans cette étude, les facteurs intervenant sur la probabilité de recevoir des transferts de fonds et discutons, à partir des données d'une enquête, leur rôle sur la population albanaise.

La migration albanaise n'est pas récente, mais elle a connu une ampleur considérable avec la chute du régime communiste d'ENVER HOXA, au début des années 1990. Les spécificités de la migration contemporaine résident dans le contexte politique et économique dans lequel elle a eu lieu, ainsi que dans son aspect massif. Au total, environ 650000 personnes, représentant $20 \%$ de la population recensée en 1989, ont quitté le pays entre 1989 et 2001 (InStituti I STATISTIKËs (INSTAT) 2004a; KING 2003). Cette migration a été marquée par une diversité des destinations (avec cependant l'Italie et la Grèce comme principaux pays d'immigration), par une proportion élevée de migrants illégaux, ainsi que par une sélection des migrants dans certaines catégories de la population. Ce sont en effet majoritairement les jeunes gens qui sont partis, modifiant par conséquent la structure démographique du pays, en provoquant à la fois un vieillissement de la population et une baisse de la population active disponible. En outre, les migrants ont été principalement recrutés parmi les plus qualifiés et les moins formés, alors que la population moyen- 
nement qualifiée présente de faibles taux d'émigration (INSTAT 2004a).

En outre, la migration internationale a été accompagnée d'une mobilité nationale importante. La concentration des migrants internes, dans quelques centres urbains le long de la côte Adriatique, a créé une division fonctionnelle du pays, séparant les territoires caractérisés par un mode de vie «traditionnel» de ceux à forte concentration d'activités économiques. Si Tirana et la côte ont bénéficié d'un développement économique important, avec cependant un marché du travail saturé, le reste du pays est caractérisé par d'anciens secteurs industriels vétustes et des terres agricoles fragmentées. Le chômage est un phénomène national, touchant $22,7 \%$ de la population active en 2001 (INSTAT 2002). Un quart de la population vit en-dessous du seuil de pauvreté, ce qui explique non seulement l'importance du secteur agricole de type familial (qui regroupe plus de la moitié des personnes actives occupées), mais aussi le développement du secteur informel, qui concerne un actif occupé sur quatre dans les régions urbaines (WORLD BANK 2003).

L'émigration a eu un impact positif sur le développement du pays, particulièrement, la forte croissance observée au milieu des années 1990 est, pour KoroviLAS (1999), attribuable aux transferts de fonds des émigrés. Cependant, Gedeshi (2002) signale le risque, pour le pays, provoqué par l'émigration, qui encourage les familles à envoyer des jeunes à l'étranger, avec comme résultat une réduction de la capacité productive du ménage.

Différentes études ont mis en évidence le lien existant entre le niveau de vie et la présence d'un membre du ménage à l'étranger, transférant des fonds (WORLD Bank 2003; United Nations Development Programme (UNDP) 2000; De Soto \& al. 2002). Or, les ménages bénéficiant d'un tel apport ne sont pas répartis uniformément sur le territoire. Par conséquent, la migration intervient non seulement à l'échelle du ménage mais aussi à l'échelle géographique, les régions comptant la proportion la plus élevée de ménages bénéficiaires étant susceptibles de voir leur économie dynamisée par l'émigration. On peut d'ailleurs s'interroger sur le rôle des transferts de fonds sur les inégalités régionales: renforcent-ils ces inégalités, au même titre que la migration, ou sont-ils un facteur de rééquilibrage? Des éléments de réponse à cette question sont présentés dans le présent article.

\section{Données et méthodes}

L'enquête sur les conditions de vie des ménages albanais (Albania living standard measurement survey -
LSMS), effectuée en 2002 auprès de 3599 ménages, est analysée dans la présente contribution (WoLD BANK \& INSTAT 2003). Réalisée par l'Office de la Statistique albanais, avec le soutien de la BANQue Mondiale, afin d'orienter les politiques économiques et sociales, cette enquête fournit des données sur les conditions de vie et la pauvreté. Le LSMS recense les différentes sources de revenus par ménage, dont les transferts de fonds. Tous les transferts privés en argent liquide et en biens depuis l'étranger sont recueillis, pour autant qu'ils aient été effectués durant les 12 derniers mois. La valeur des biens, estimée par l'interviewé, a été additionnée aux apports financiers pour former le montant total des remittances par ménage.

La présence à l'étranger d'un membre du ménage ne peut être que partiellement identifiée à partir de ces données. Les migrants internationaux sont uniquement recensés lorsque leur mère est encore en vie et présente dans le ménage. Par conséquent, pour un quart des bénéficiaires de remittances recensés par le LSMS, il n'est pas possible d'identifier un migrant quelconque. L'analyse portera pour cette raison sur l'ensemble des ménages albanais.

La variable au centre de cette étude est donc le fait de bénéficier ou non de transferts de fonds provenant d'un migrant privé établi à l'étranger. Cette information sera mise en relation, à l'aide d'un modèle statistique, avec divers facteurs indépendants traduisant la position des ménages dans l'échelle sociale de l'Albanie. La méthode utilisée, la régression logistique, mesure l'impact de ces facteurs explicatifs sur la probabilité de recevoir des fonds (cf. Cox \& SNELl 1989). Les variables explicatives retenues dans le modèle sont:

- le type de ménage, en cinq modalités: ménage individuel, couple, ménage monoparental dirigé par une femme, ménage monoparental dirigé par un homme et ménage nucléaire, ménage étendu;

- le niveau de formation du chef de ménage exprimé par le nombre d'années scolaires suivies (jusqu'à 4 ans, entre 5 et 8 ans) et par la formation atteinte (formation secondaire ou universitaire);

- le statut d'activité du chef de ménage selon les modalités suivantes: aux âges de la retraite (60 ans et plus), inactif, actif occupé dans une entreprise non agricole indépendante, autre actif occupé, au chômage;

- le statut d'activité des autres membres du ménage: aucun membre n'est en âge d'exercer une activité économique, membres tous inactifs, au moins un membre actif dans une entreprise non agricole indépendante, une majorité de membres est active occupée, une majorité de membres est au chômage;

- l'histoire migratoire interne du ménage en trois modalités: ménage sans migration interne, fonda- 
tion ou recomposition d'un ménage après une migration interne du chef de ménage (et éventuellement ses enfants) ou par l'arrivée d'un membre migrant, autres migrations internes des membres du ménage;

- la situation de pauvreté relative. On a considéré, pour mesurer cet indicateur, la distribution des revenus mensuels courants auto-déclarés des ménages dans la préfecture et le lieu de résidence (urbain/ rural). On a ensuite considéré comme ménages victimes de la pauvreté ceux dont le revenu mensuel par tête est situé dans le premier quartile de cette distribution;

- le lieu de résidence et la propriété terrienne en trois modalités: vit en milieu urbain, vit en milieu rural avec propriété agricole, vit en milieu rural sans propriété agricole;

- enfin, la préfecture de résidence. L'Albanie est divisée en 12 préfectures.

\section{Résultats}

\subsection{Importance des transferts de fonds}

Le tableau 1 fournit une première estimation de l'importance des transferts de fonds parmi les personnes enquêtées. Au total, $23 \%$ des ménages déclarent recevoir des fonds de la part d'un migrant (Tab. 1). Si l'on excepte la préfecture de Tirana, qui présente une très faible proportion de ménages bénéficiaires, la part de ces ménages est la même quelle que soit la région de résidence (urbain/rural). La médiane des montants reçus par ménage bénéficiaire s'élève à 700 dollars pour l'ensemble du pays, ce qui représente $44 \%$ du revenu annuel estimé à partir du revenu mensuel courant du ménage. Les valeurs des $1^{\text {er }}$ et $3^{\text {e }}$ quartiles sont de 280 et 1400 dollars, montrant la grande diversité des situations. La distribution des remittances est fortement dissymétrique avec une tendance vers des valeurs élevées.

Les montants reçus sont plus élevés dans les régions rurales que dans les régions urbaines, tant en termes absolus (montant en dollars) que relatifs (montant ramené au revenu annuel). Ce fait s'explique sans doute par le nombre moins élevé de donneurs potentiels dans les ménages urbains, ainsi que, peut-être, par des stratégies d'envois différentes parmi les migrants en provenance de zones urbaines (Photos 1 et 2).

\subsection{La géographie des transferts de fonds}

L'application du modèle de régression logistique fournit une lecture de l'incidence des transferts de fonds en fonction de la préfecture de domicile, après prise en compte des autres variables incluses dans le modèle. La préfecture côtière de Fier a été considérée comme préfecture de référence. Les odds ratios fournis par le modèle informent sur les positions préfectorales en matière de remittances. Les préfectures bénéficiaires des

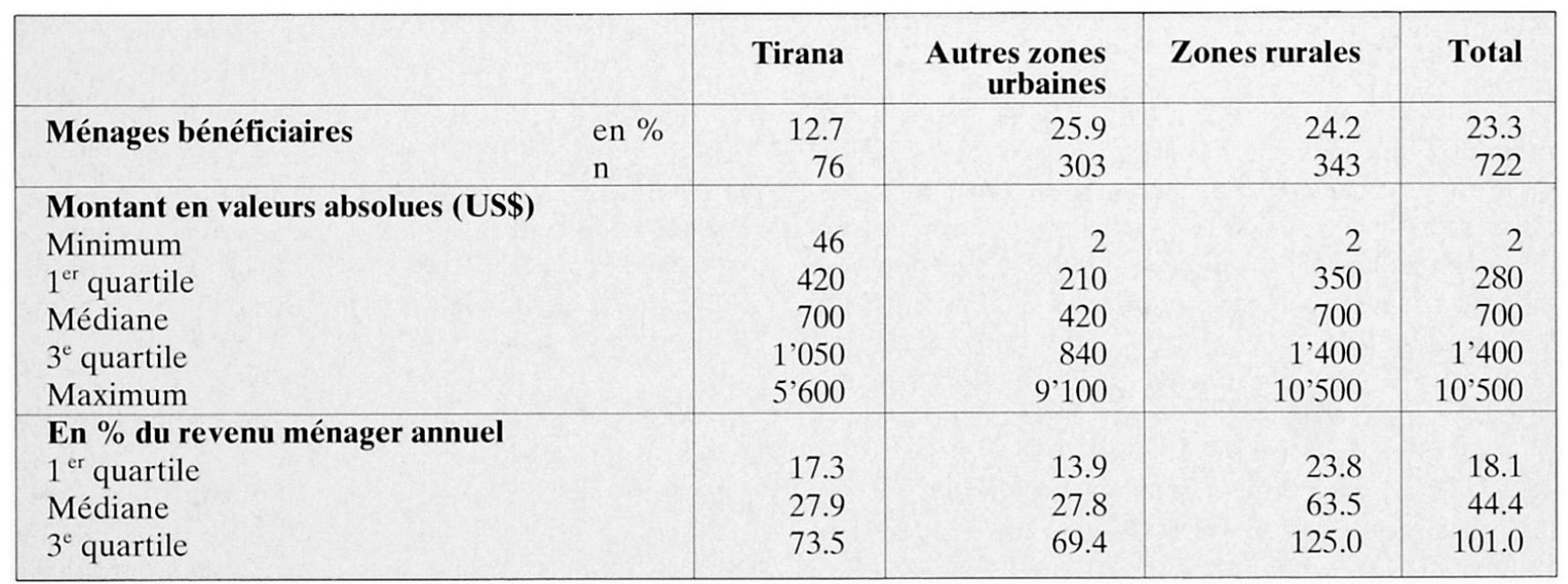

Le revenu ménager annuel a été extrapolé à partir du revenu ménager mensuel courant auto-déclaré.

Tab. 1: Distribution en quartiles des transferts de fonds (en dollars et en $\%$ du revenu ménager annuel), selon le lieu de résidence, Albanie 2002

Verteilung der Geldtransfers (in Dollar und in \% des jährlichen Haushaltseinkommens, nach Wohnort, in Albanien (2002); Abgrenzung nach Quartilen

Quartile distribution of remittances (in dollars and in \% of the monthly household income) by type of settlement, Albania 2002

Source: Albania living standard measurement survey 2002 

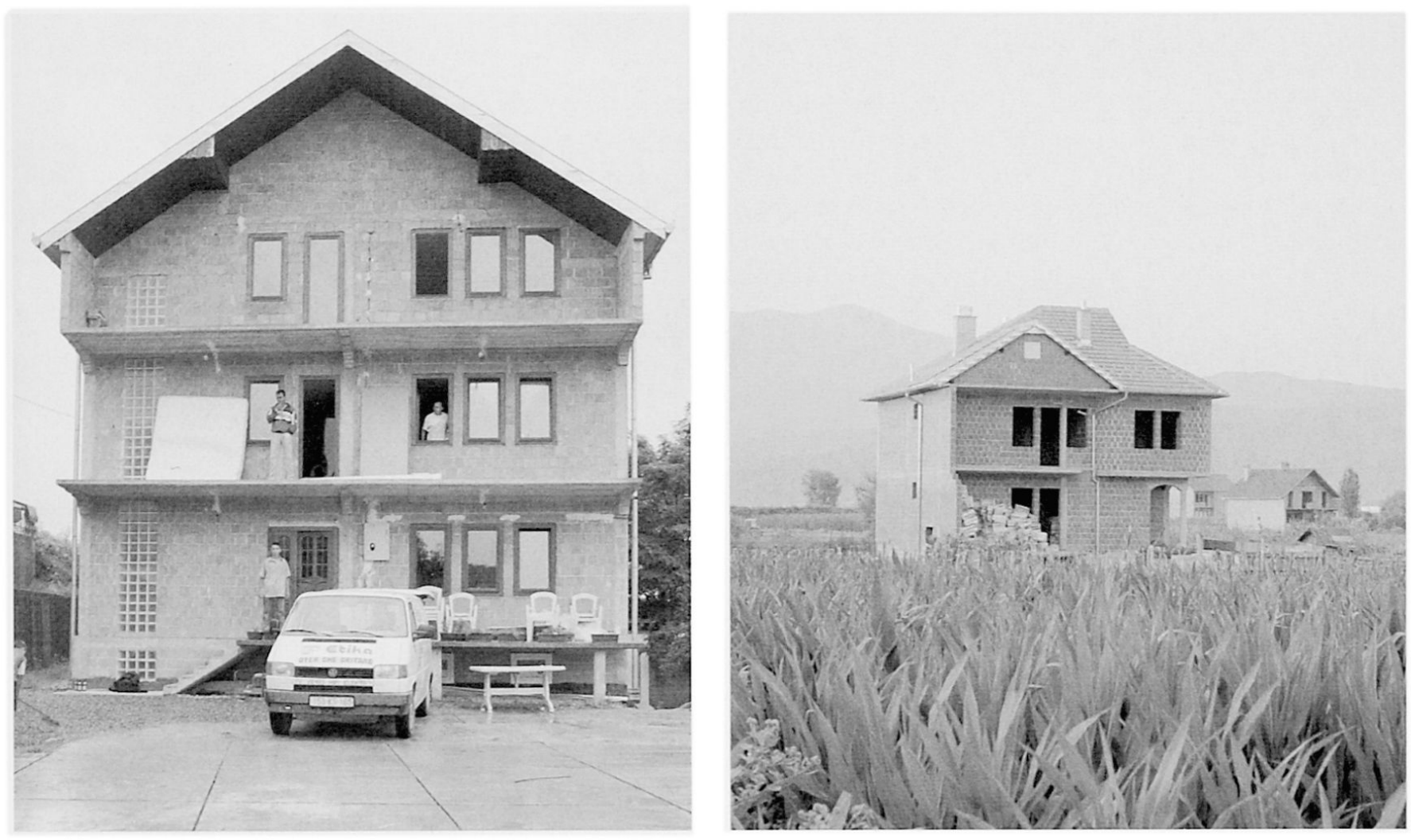

Photos 1 et 2: Nouvelles constructions au Kosovo: L'immigration n'est pas sans retours occasionnels. Investissements immobiliers de migrants résidant en Suisse

Neubauten im Kosovo: Immigration verläuft nicht ohne gelegentliche Rückkehr. Immobilieninvestitionen von in der Schweiz lebenden MigrantInnen

Construction sites in Kosovo: Immigration often involves occasional return. Property investments of migrants living in Switzerland

Photos: M. RoD

transferts de fonds sont en premier lieu situées le long de la côte Adriatique et se caractérisent par une importance considérable des flux de migration interne (Skhoder, Lezhe, Durres ou Vlore, cf. Tab. 2). Ces préfectures, davantage urbanisées, se caractérisent, selon le LSMS, par un marché du travail dual et un important entreprenariat. Berat, localisée dans le Sud de l'Albanie, représente également un pôle de réception des transferts de fonds. La probabilité de bénéficier de transferts de fonds est par contre faible pour les ménages résidant dans les préfectures périphériques ou situées à l'intérieur du pays, préfectures davantage agricoles et touchées par l'exode rural et la migration vers les centres économiques. La préfecture de Kukes, située dans le Nord-Est du pays, illustre cette situation. Cette région a souffert d'une forte émigration familiale à destination vers le reste du pays (INSTAT 2004a), ce qui limite l'arrivée de fonds provenant de l'étranger. Pour sa part, en dépit de son importance dans la géographie de la migration tant interne qu'internationale, la préfecture-capitale, Tirana, est relativement peu propice à la réception de transferts de fonds, ce qui confirme l'observation issue du tableau 1. Cette particularité peut s'expliquer par le fait que Tirana est avant tout une région d'arrivée des migrations internes provenant des régions pauvres du Nord-Est du pays (INSTAT 2004a), et ne fournit pas, contrairement aux régions côtières, une proportion élevée d'émigrants fournisseurs de remittances.

On observe également que les ménages vivant dans les zones rurales - et particulièrement ceux qui ne possèdent pas de terres agricoles - montrent un risque accru en ce qui concerne le bénéfice de transferts de fonds par rapport aux ménages urbains. Ces résultats s'expliquent par le fait que le partage des terres, lors de la «décollectivisation», a donné lieu à des conflits entre les nouveaux arrivants issus de la migration interne et les anciens résidents. Les premiers, souvent exclus du processus de distribution des terres (Lemel 1998), dépendent fortement de fonds provenant de l'étranger. On peut suggérer que ces fonds serviront, un jour ou l'autre, à financer l'achat de terre, achat nécessaire pour atteindre une qualité de vie acceptable en milieu rural (DE Soto \& al. 2002). 


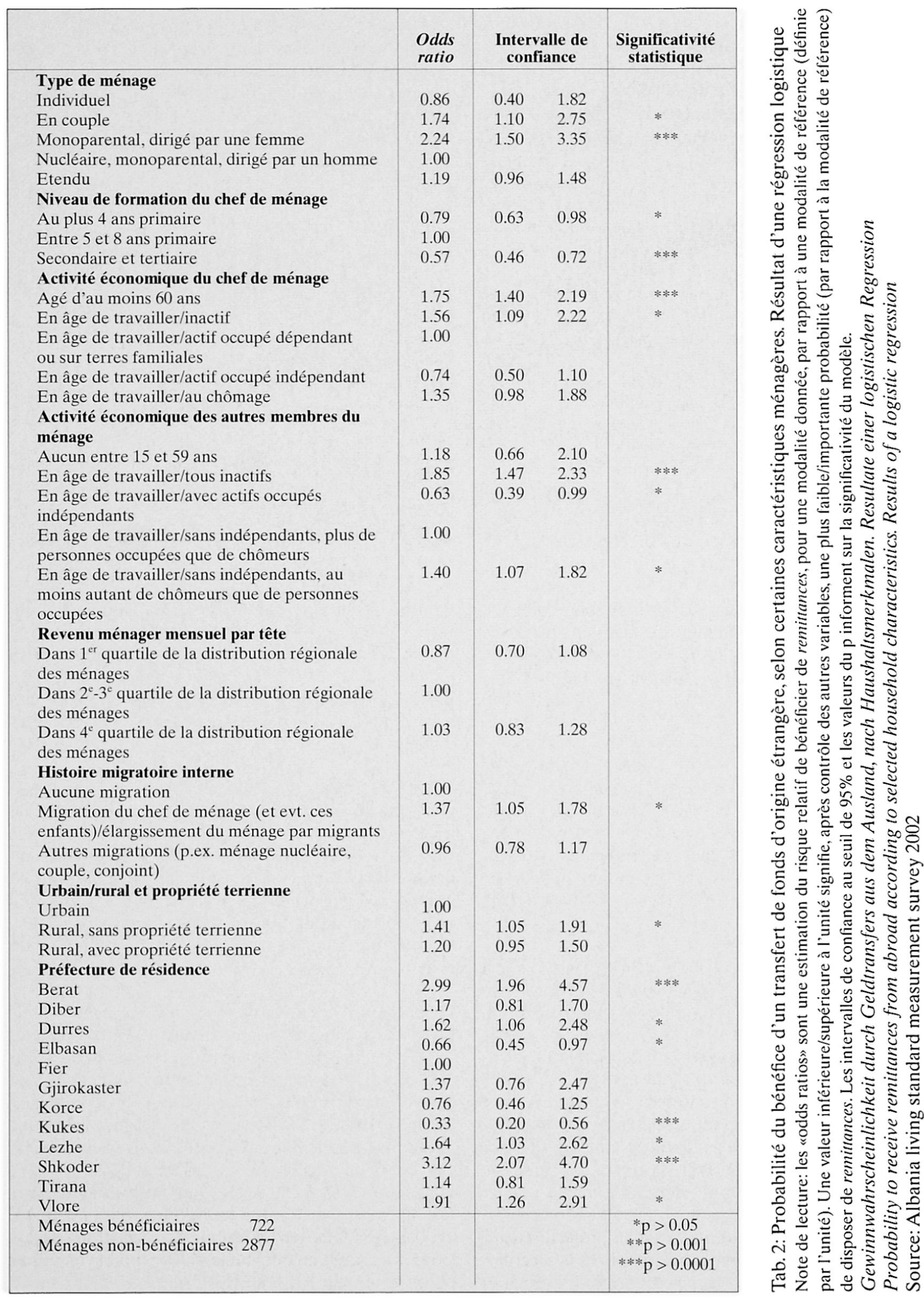




\subsection{Les facteurs familiaux déterminant la probabilité du bénéfice de transferts de fonds}

Le risque significativement élevé, par rapport aux ménages nucléaires, de recevoir des remittances pour les ménages monoparentaux dirigés par une femme ressort en outre clairement du modèle (Tab. 2). Il s'explique bien évidemment par le fait que la migration peut être le motif du caractère monoparental du ménage, caractère alors le plus souvent provisoire. Les ménages vivant en couple sont également concernés par le phénomène, en particulier lorsqu'ils arrivent en fin de vie, et lorsque les enfants sont adultes. L'émigration d'un enfant du ménage soulage alors la charge financière et, par le biais de la solidarité intergénérationnelle, peut favoriser les transferts financiers en Albanie (GEDEsHi \& al. 2003). Les remittances jouent ainsi un rôle de soutien face au handicap économique caractérisant les personnes âgées. Dans une certaine mesure, ce soutien international a remplacé le ménage étendu dans sa fonction d'entraide.

Le capital humain du chef de ménage constitue un autre facteur influençant la probabilité du bénéfice de remittances. Les ménages à capital humain très faible ou élevé ont une probabilité diminuée de pouvoir bénéficier de transferts de fonds par rapport aux ménages à capital humain médian. De plus, les ménages dirigés par une personne inactive présentent un risque significativement accru de percevoir des fonds, par rapport à ceux qui sont dirigés par une personne salariée. Les ménages dirigés par un chômeur présentent un risque également élevé, quoique non significatif. Le fait de bénéficier de remittances pallie probablement à la pauvreté observée dans les ménages de chômeurs et d'inactifs, avérée par la banque mondiale (WORLD BANK 2003). En ce qui concerne les autres membres du ménage, des résultats similaires s'observent avec un risque plus élevé de bénéficier de fonds lorsque ces membres sont inactifs ou au chômage, et un risque plus faible lorsqu'ils sont insérés au marché du travail.

Contrairement à ce que l'on pourrait penser à la lecture de Kule et al. (2002), on ne détecte pas un important dynamisme entrepreneurial parmi les bénéficiaires. Ainsi, les ménages, ayant fondé une entreprise ménagère non agricole, ont une faible probabilité de pouvoir bénéficier des transferts de fonds. Si le fait de monter sa propre entreprise équivaut à créer son propre emploi, comme cela semble avéré pour la quasi-totalité des travailleurs du secteur informel (World BANK 2003), on peut suggérer qu'en cas de remittances, la nécessité de créer son propre emploi est moins pressante. En outre, le manque d'occasions économiques est une raison fréquente pour l'émigration (GEDESHl et al. 2003) et il est probable que les migrants établis à l'étranger sont «sélectionnés» au sein des ménages qui, pour des raisons diverses, n'ont pas pu ou voulu créer une entreprise individuelle. Les résultats obtenus concernant les indépendants suggèrent par ailleurs que les bénéficiaires de remittances utilisent les fonds reçus à des usages autres que la création d'entreprises: p.ex. dans la sphère privée, l'équipement du ménage, l'épargne (DE RAPPER et al. 2000).

L'histoire migratoire interne du ménage a également son importance, comme le montre le risque significativement plus élevé, par rapport aux ménages n'ayant pas vécu de migration, de bénéficier de remittances pour les ménages formés ou recomposés par un migrant interne (personne de référence ou autre membre).

Enfin, on ne constate pas de probabilité différenciée de recevoir des transferts de fonds en fonction de la position des ménages sur l'échelle des revenus.

\section{Discussion}

Dans la présente étude, nous avons discuté, à l'aide d'un modèle, des différents facteurs intervenant en faveur de la disponibilité de fonds en provenance de l'étranger, parmi les ménages albanais. Les résultats obtenus montrent l'importance du phénomène qui est à relier à la forte émigration albanaise observée depuis les années 1990. Non seulement le quart des ménages albanais bénéficient de remittances, mais en outre les montants reçus représentent une part très importante, souvent même majoritaire, du revenu total du ménage.

Cette situation n'a pas seulement pour conséquence de créer une dépendance partielle d'un certain nombre de ménages par rapport à des fonds étrangers, mais interroge sur l'utilisation des fonds disponibles dans une optique de développement économique et social. A ce propos, quelques résultats observés peuvent être discutés plus en détail. Au préalable, il convient d'émettre quelques remarques utiles à l'interprétation de ceux-ci.

D'une part, il convient de noter que les données utilisées dans cette étude proviennent d'une enquête, qu'elles sont donc soumises à certaines limites. En particulier, les remittances sont appréhendées par une auto-déclaration de la part de la personne enquêtée, ce qui les expose à d'éventuelles inexactitudes. D'autre part, ainsi que cela a été signalé, nous ne disposons pas systématiquement d'une information sur la présence de proches à l'étranger. Dans notre modèle, nous testons la probabilité de recevoir des fonds en considérant ensemble deux sous-populations, celles disposant d'un enfant à l'étranger et celles ne disposant pas d'un tel réseau. En d'autres termes, les données ne 
permettent pas de mesurer exactement qui, parmi les ménages dont l'un des membres vit à l'étranger, reçoit des fonds, et qui n'en reçoit pas. Or, seules de telles informations permettent de décrire parfaitement les stratégies familiales en la matière.

Nonobstant ces limites, les analyses montrent différents résultats pouvant être mis en relief par rapport à la littérature internationale. En particulier, le fait que les ménages composés d'un couple âgé aient été identifiés comme bénéficiaires, rejoint les observations d'Itzigsohn (1995) et de SANA (2003) dans d'autres pays. Ce résultat semble démontrer le caractère intergénérationnel des transferts (de l'enfant aux parents) et le fait que ceux-ci ne touchent pas seulement des entrepreneurs potentiels, mais des personnes hors du marché du travail. La sous-représentation des personnes âgées parmi les pauvres (INSTAT 2004b) est à ce propos un indicateur du rôle des remittances dans la lutte contre la pauvreté. Conformément à ce qui a été observé dans d'autres pays, cette étude montre en outre que le soutien économique depuis l'étranger touche en premier lieu les ménages se trouvant dans des situations professionnelles précaires (inactivité ou chômage). Les remittances peuvent par ailleurs permettre de rester hors du marché du travail (par exemple pour les femmes ayant des charges familiales), résultat qui avait déjà été observé dans d'autres contextes (ItZigsohn 1995; Russel 1986; LuCAS \& STARK 1985). La relation entre les transferts de fonds et le développement de petites et moyennes entreprises ménagères, souvent avancée comme un aspect positif des remittances pour le développement (DuRAND et al. 1996), n'a, par contre, pas pu être démontrée dans cette étude.

La littérature internationale ne fournit pas d'avis tranchés sur le rôle du contexte de vie sur la réception de fonds. Pour certains, les ménages urbains, mieux formés et donc plus susceptibles d'avoir des membres à l'étranger, bénéficient davantage de remittances que les ménages ruraux (Rodriguez 1996). Pour d'autres, le différentiel de revenu entre villes et campagnes pourrait expliquer pourquoi les milieux ruraux sont les principaux bénéficiaires de fonds (JoNES 1998). En Albanie, les régions rurales sont les principales bénéficiaires, constat que nous pouvons mettre en relation avec la motivation d'acquisition des terres.

Plus généralement, la localisation des ménages bénéficiaires joue un rôle important dans la structure économique du pays et fournit des éléments d'information sur le rôle des migrations dans le développement régional. SKELDON (2003) suggère que la migration peut parfois se dérouler par étape («step by step migration»), avec une première migration interne du ménage en direction des centres et, plus tard, une seconde migration d'une partie du ménage vers l'étranger. Ce schéma semble confirmé en Albanie (cf. INSTAT 2004a). Compte tenu des différentiels géographiques dans les remittances, ce fait conduit à un accroissement des ressources dans les régions côtières, zones d'immigration interne, où se retrouvent les candidats au départ.

\section{Bilan}

L'importante émigration et les transferts de fonds qui en résultent ont, sans doute, contribué à la croissance économique albanaise. Les résultats de la présente étude suggèrent que la fonction première des transferts de fonds réside avant tout dans l'allégement de la pauvreté à l'échelle des ménages, mais que ces transferts peuvent contribuer aux différentiels de développement économique à l'échelle des régions. Les remittances sont davantage envoyées vers des ménages potentiellement pauvres, et contribuent donc à l'élévation des conditions de vie des ménages les plus défavorisés. Ayant souvent procédé à des stratégies migratoires complexes, ces ménages résident dans les zones économiques centrales du pays mais n'ont pas (encore) réussi à s'insérer au marché du travail.

\section{Bibliographie}

Cox, R.D. \& E.J. SNELl (1989): The analysis of binary data. $-2^{\text {nd }}$ edition, London: Chapman and Hall.

De Rapper, G., Deslondes, O. \& M. Roux (2000): Dimanche à Miras, lundi à Dipotamia - La frontière albano-grecque dans la région de Bilisht et de Kastoria. - In: Cahiers d'études sur la Méditerranée orientale et le monde turco-iranien 29: 199-223.

De Soto, H., Gordon, P., Gedeshi, I. et. al. (2002): Poverty in Albania - a qualitative assessment. - Technical paper No. 520, Europe and Central Asia environmentally and socially sustainable development series, Washington: The World Bank: 131.

Durand, J., Kandel, W., Parado, E.A. et al. (1996): International migration and development in Mexican communities. - In: Demography 33, 2: 249-264.

GAMmELtopf, P. (2002): Remittances and other financial flows to developing countries. - Working paper 02.11, Centre for Development Research, Copenhagen: 25.

Gedeshi, I. (2002): Role of remittances from Albanian emigrants and their influence in the country's economy. - In: Eastern European economics 40, 5: 49-72.

Gedeshi, I., Mara, H. \& P. Shilda (2003) : The encouragement of social-economic development in relation to the growth of the role of the remittances. - Research report commissioned by UNDP and Soros Foundation, Tirana: 72.

INSTITUTI I STATISTIKËs (INSTAT) (2002): The population of Albania in 2001. - Tirana: INSTAT: 87. 
InSTITUTI I STATISTIKËs (INSTAT) (2004a): Migration in Albania. - Tirana: INSTAT: 64.

INSTITUTI I STATISTIKËS (INSTAT) (2004b): Living conditions and inequality in Albania. - Tirana: INSTAT: 47.

ItZigsohn, J. (1995): Migrant remittances, labor markets, and household strategies: a comparative analysis of low-income household strategies in the Caribbean basin. - In: Social forces 74, 2: 633-655.

JONES, R.C. (1998): Remittances and inequality: a question of migration stage and geographic scale. - In: Economic geography $74,1: 8-25$.

KING, R. (2003): Across the sea and over the mountains: documenting Albanian migration. - In: Scottish geographical journal 119, 3: 283-309.

Koravilas, J.P. (1999): The Albanian economy in transition: the role of remittances and pyramid investment schemes. - In: Post-communist economies 11, 3: 399-415.

Kule, D., Mancellari, A., Papanagos, H. et al. (2002): The causes and consequences of Albanian emigration during transition: evidence from micro data. - In: International migration review 26, 1:229-239.

Lemel, H. (1998): Rural land privatisation and distribution in Albania: evidence from the field. - In: Europe-Asia studies 50,1:121-140.

LuCAS, R.E.B. \& O. STARK (1985): Motivations to remit: evidence from Botswana. - In: Journal of political economy 93, 5: 901-918.

RATHA, D. (2004): Understanding the importance of remittances. - In: Migration information source, www.migrationinformation.org (1.10.2004).

RODRIGUEZ, E.R.(1996): International migrants' remittances in the Philippines. - In: The Canadian journal of economics 29, special issue 2: 427-432.

Russel, S.S. (1986): Remittances from international migration: a review in perspective. - In: World development 14, 6: 677-696.

SANA, M. (2003): Household composition, family migration and community context: migrant remittances in four countries. - Paper presented at the 2003 meeting of the Latin American Studies Association, March 27-29, Dallas, Texas.

SKELDON, R. (2003): Interlinkages between internal and international migration and development in the Asian region. - Paper presented at the Ad Hoc Expert Group Meeting on Migration and Development, August 27-29, Bangkok.

UruçI, E. \& I. Gedeshi (2003): Remittances management in Albania. - Working paper 3/2003, Centro Studi di Politica Internationale, Roma: 33.

United Nations Development Programme (UNDP) (2000): Albanian human development report. - Tirana: UNDP: 69.

WORLD BANK (2003): Albanian poverty assessment. Report No. 26213-AL, Human Development Sector, Unit Europe and Central Asia Region, World Bank: 141.
WORLD BANK \& INSTAT (2003): Albania living standard measurement survey. - Basic information document: 69.

\section{Résumé: Les facteurs déterminants de la réception de transferts de fonds des migrants et leur impact sur la pauvreté en Albanie}

La présente étude identifie les facteurs qui déterminent la réception de transferts de fonds de migrants en Albanie, afin de fournir des informations quant à leur impact sur le niveau de vie des ménages. Si un cinquième de la population albanaise a quitté le pays depuis 1989 , les transferts de fonds ont représenté durant cette même période jusqu'à $20 \%$ du Produit National Brut (PNB) et, en terme de valeur médiane, $44 \%$ du revenu ménager des bénéficiaires. Comme le montrent les résultats d'une régression logistique appliquée sur les données d'une enquête (Albania living standard measurement survey), effectuée en 2002, cet apport financier se concentre dans les régions économiquement développées drainant les flux de migration interne, à savoir la région côtière. Or, les transferts de fonds bénéficient le plus aux couches inférieures de l'échelle sociale régionale. En effet, les ménages bénéficiaires sont caractérisés par un handicap tant économique que social. Les transferts de fonds en Albanie semblent donc renforcer les disparités de richesses entre les régions, mais jouent un rôle actif dans la réduction des inégalités inter-familiales de revenu dans les espaces centraux et atténuent la pauvreté.

\section{Zusammenfassung: Die Determinanten des Erhalts von Geldüberweisungen von MigrantInnen und die Auswirkungen auf die Armut in Albanien}

Diese Studie analysiert die Determinanten des Erhalts bzw. Nichterhalts von Geldüberweisungen in Albanien. Ziel ist es, Informationen über deren Einfluss auf das Lebensniveau der Haushalte zu sammeln. Seit 1989 hat ein Fünftel der Bevölkerung das Land verlassen und durch Geldüberweisungen $\mathrm{zu}$ annähernd $20 \%$ des Bruttonationalprodukts beigetragen. Diese Transfers machen im Mittel $44 \%$ des Einkommens der betreffenden Haushalte aus. Die Resultate der logistischen Regression, welche auf die im Jahre 2002 gesammelten Umfragedaten (Albania living standard measurement survey) angewendet wurde, zeigen, dass die Haushalte an der Adriatischen Küste, die sich durch eine höhere wirtschaftliche Entwicklung auszeichnet, am häufigsten von diesen Transfers profitieren. Obwohl die begünstigten Haushalte sich in den unteren Gesellschaftsschichten befinden, die Geldtransfers daher interfamiliäre Unterschiede der Lebensstandards nivellieren helfen, kann eine Verstärkung der interregionalen Disparitäten aufgezeigt werden. 


\section{Abstract: Determinants of the reception of migrant remittances and their impact on poverty in Albania} This paper analyses the factors that determine the receipt or non-receipt of migrant remittances to Albania in order to gain a better understanding of the impact of such monies on the living standard in affected areas. While one fifth of the Albanian population has left the country since 1989 , the country as a whole has benefited from remittances which make up at times $20 \%$ of the Gross Domestic Product (GPD) and $44 \%$ of the household income of the recipients. The results of a logistic regression applied on survey data collected in 2002 indicate that those households on the Adriatic Coast that are characterized by a higher economic development, benefit most from these transfers. Despite the fact that most of the recipient households are in the lower classes and thus economically and socially disadvantaged, the remittances appear to contribute more towards a levelling out of inter-family wealth disparity, rather than to a softening of wealth disparity at an interregional level.

Mathias Lerch, Géographe diplômé, Dr. Philippe Wanner, Forum suisse pour l'étude des migrations et de la population, Rue St. Honoré 2, CH-2000 Neuchâtel. e-mail:

Mathias.Lerch@unine.ch

Philippe.Wanner@unine.ch

Manuskripteingang/received/manuscrit entré le 13.4.2005

Annahme zum Druck/accepted for publication/accepté pour l'impression: 9.9.2005 